\title{
Atomic Monolayer Deposition on the Surface of Nanotube Mechanical Resonators
}

\author{
A. Tavernarakis, ${ }^{1}$ J. Chaste, ${ }^{2, *}$ A. Eichler, ${ }^{1,2, \dagger}$ G. Ceballos, ${ }^{2}$ M. C. Gordillo, ${ }^{3}$ J. Boronat, ${ }^{4}$ and A. Bachtold ${ }^{1,2}$ \\ ${ }^{1}$ ICFO-Institut de Ciencies Fotoniques, Mediterranean Technology Park, 08860 Castelldefels, Barcelona, Spain \\ ${ }^{2}$ Institut Català de Nanotecnologia, Campus de la UAB, E-08193 Bellaterra, Spain \\ ${ }^{3}$ Departamento de Sistemas Físicos, Químicos y Naturales, Universidad Pablo de Olavide, \\ Carretera de Utrera, km 1, E-41013 Sevilla, Spain \\ ${ }^{4}$ Departament de Física i Enginyeria Nuclear, Universitat Politècnica de Catalunya, \\ B4-B5 Campus Nord, 08034 Barcelona, Spain
}

(Received 24 January 2014; published 14 May 2014)

\begin{abstract}
We study monolayers of noble gas atoms ( $\mathrm{Xe}, \mathrm{Kr}$, Ar, and $\mathrm{Ne}$ ) deposited on individual ultraclean suspended nanotubes. For this, we record the resonance frequency of the mechanical motion of the nanotube, since it provides a direct measure of the coverage. The latter is the number of adsorbed atoms divided by the number of the carbon atoms of the suspended nanotube. Monolayers form when the temperature is lowered in a constant pressure of noble gas atoms. The coverage of Xe monolayers remains constant at $1 / 6$ over a large temperature range. This finding reveals that Xe monolayers are solid phases with a triangular atomic arrangement, and are commensurate with the underlying carbon nanotube. By comparing our measurements to theoretical calculations, we identify the phases of Ar and Ne monolayers as fluids, and we tentatively describe $\mathrm{Kr}$ monolayers as solid phases. These results underscore that mechanical resonators made from single nanotubes are excellent probes for surface science.
\end{abstract}

Carbon nanotubes have motivated considerable research effort for the study of gas adsorption onto substrates that approach the one-dimensional limit [1-9]. Many studies have been carried out on mats and films of nanotube bundles, but the interpretation of those measurements is complicated by the fact that the binding energy of the gas atoms on the substrate is not homogeneous. That is, the binding energy depends on whether the atom is located on an individual nanotube, at the junction between two crossing nanotubes, or along the interstitial channel formed between two parallel nanotubes. Recently, this homogeneity problem was solved by studying gas adsorption on individual nanotubes, a technical feat considering the tiny amount of adsorbed atoms [7-9]. For this, nanotubes were employed both as substrates for adsorption and as detectors. Namely, the nanotubes were operated as mechanical resonators, the resonance frequency being exquisitely sensitive to the number of adsorbed atoms [9-12].

Atoms adsorbed on graphitic surfaces can form a rich variety of different phases, such as vapor, liquid, supercritical fluids, and solids [13]. The solid phase can be either commensurate or incommensurate with the graphene surface [Figs. 1(a), 1(b)]. The commensurate solid phase is robust, since the crystal formed by the adsorbed atoms is strongly pinned to the underlying carbon surface. Commensurate monolayers on graphite feature a well defined ratio between the number of adsorbed atoms and the number of carbon atoms at the surface. This ratio, called coverage, is often $1 / 6$ for noble gas atoms, which corresponds to a registered $\sqrt{3} \times \sqrt{3}$ lattice (Ref. [13],
Table 6.1). This particular coverage value arises because, in this solid phase, noble gas atoms form a two-dimensional triangular arrangement in which atoms occupy the center of carbon hexagons, leaving an empty one in the center of the triangle [see Fig. 1(b)]. Nanotubes are also expected to host commensurate solids; however, due to cylindrical boundary conditions, these solids exist only for some specific nanotube chiralities $(n, m)$, namely when $(n-m) / 3$ is an integer [7]. Remarkably, this is also the condition for nanotubes to be metallic.

Solid and fluid monolayers made of noble gas atoms, such as $\mathrm{Xe}, \mathrm{Kr}$, and $\mathrm{Ar}$, were measured on graphite surfaces only when the coverage was comparable to or larger than 1/6 (Ref. [13], Chap. 6). When adsorbed on a nanotube surface, the coverage of incommensurate solids and fluids is expected to become larger than that measured on graphite due to the curvature of the nanotube [8]. This is because (i) adsorbed atoms form cylindrical monolayers with a surface larger than that of the carbon nanotube, and (ii) the two-dimensional density of noble gas atoms is, to a good approximation, independent of the curvature of the monolayer. Recently, monolayers of $\mathrm{Kr}$ and Ar were obtained on individual nanotubes by increasing the pressure of $\mathrm{Kr}$ or $\mathrm{Ar}$ gas surrounding the nanotube [7]. These monolayers were identified as solids, but these phases were fragile, since the number of atoms in the monolayer was very sensitive to temperature.

Here, we report on the formation of monolayers of Xe, $\mathrm{Kr}$, $\mathrm{Ar}$, and $\mathrm{Ne}$ on individual ultraclean nanotubes upon decreasing temperature. The pressure was kept constant, typically in the $10^{-7}$ mbar range. We prepared the nanotube 
(a)

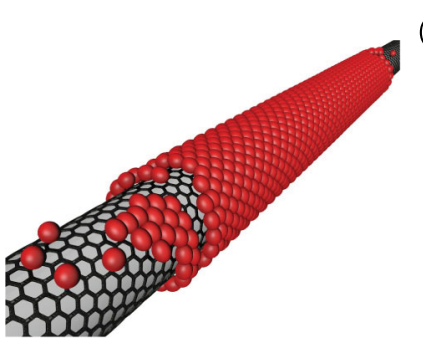

(b)

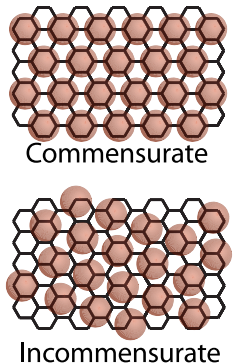

(c)

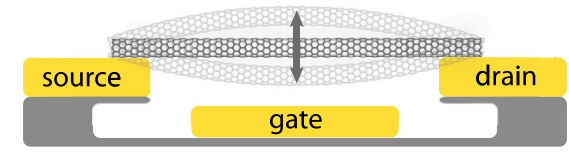

(d)

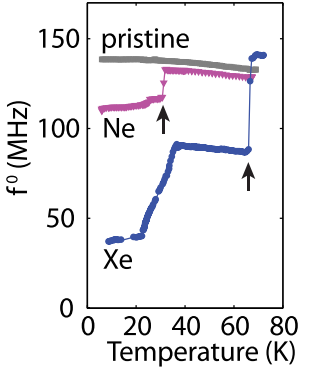

(e)

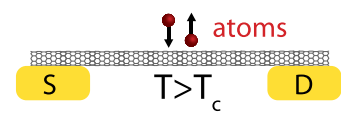

(f)

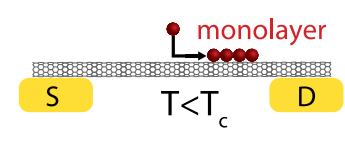

FIG. 1 (color online). (a) Growth of an atomic monolayer on a nanotube. (b) Schematics of monolayers in the solid phase that are commensurate (top) and incommensurate (bottom) with the carbon substrate. The adsorbed atoms are represented by red spheres, whereas the carbon surface is depicted by the honeycomb lattice. (c) Layout of the nanotube resonator. (d) Resonance frequency upon lowering temperature while dosing Xe and $\mathrm{Ne}$ using a pinhole micromanipulator. The curve labeled "pristine" corresponds to the $T$ dependence of $f^{0}$ when we do not dose atoms. The pressure is $3 \times 10^{-7}$ mbar for the $\mathrm{Xe}$ and the $\mathrm{Ne}$ measurements and $3 \times 10^{-11}$ mbar for the pristine measurement. (e),(f) Schematics showing the balance of atoms impinging on and departing from the nanotube above and below the characteristic temperature $T_{c}$.

by thoroughly current annealing it in order to remove contamination from the surface. The monolayer of Xe was found to be the most robust phase. Its coverage remained constant at $1 / 6$ over a large temperature range, indicating the formation of a $\sqrt{3} \times \sqrt{3}$ commensurate solid. The coverages of the monolayers made from $\mathrm{Kr}$, Ar, and $\mathrm{Ne}$ were less stable against temperature variations. We compare our experimental findings to theoretical calculations in order to establish the nature of these phases.

In order to demonstrate commensurate solid phases, we fabricated resonators based on ultraclean nanotubes that are metallic. For this, we used the fabrication process that we described in Ref. [14]. As shown in Fig. 1(c), the nanotube is contacted by two electrodes and is suspended over a trench with a gate electrode at the bottom. The nanotube was grown by chemical vapor deposition in the last step of the fabrication process in order to reduce contamination
[15] (Supplemental Material, Sec. I [16]). The measurement of the electrical conductance as a function of the voltage applied to the gate electrode allowed us to select nanotubes that are metallic with a small energy gap (Supplemental Material, Sec. III [16]).

The mechanical motion was driven and detected using the frequency-modulation mixing technique [23] (Supplemental Material, Sec. III [16]). We carried out the experiment in a home-built ultrahigh vacuum cryostat that reaches a base pressure of $\sim 3 \times 10^{-11}$ mbar. The nanotube was cleaned by current annealing. Noble gas atoms were dosed from a room-temperature supply with a pinhole microdoser. We studied three nanotubes yielding similar results. We discuss, in the following, the data for one device. Data for a second device are shown in the Supplemental Material, Sec. VIII [16].

Monolayers of noble gas atoms formed on the nanotube when the temperature $(T)$ was lowered while keeping a constant pressure of noble gas in the cryostat chamber. The formation was monitored by measuring the resonance frequency $f^{0}$ (that is, by continuously recording the response of the nanotube resonator to the driving frequency). Figure 1(d) shows prominent jumps of $f^{0}$ to lower frequencies upon lowering $T$ (see arrows), indicating the sudden adsorption of a large quantity of atoms onto the nanotube. For comparison, when we did not dose atoms, the temperature dependence of $f^{0}$ is weak and monotonic [gray curve labeled "pristine" in Fig. 1(d)]. This weak dependence is attributed to the thermal expansion of the electrodes which modifies the spring constant of the nanotube resonator [24]. The coverage at $T$ is extracted using

$$
\varphi(T)=\frac{N_{\mathrm{ads}}(T)}{N_{\mathrm{c}}}=\frac{m_{\mathrm{c}}}{m_{\mathrm{ads}}}\left[A\left(\frac{f_{\mathrm{prist}}^{0}(T)}{f_{\mathrm{ads}}^{0}(T)}\right)^{2}-1\right],
$$

where $N_{\mathrm{c}}$ is the number of $\mathrm{C}$ atoms of the suspended nanotube, $N_{\text {ads }}$ is the number of adsorbed atoms on the nanotube, and $m_{\mathrm{c}}$ and $m_{\mathrm{ads}}$ are the atomic masses of carbon and adsorbed atoms, respectively. Here, $f_{\text {ads }}^{0}$ is the resonance frequency when dosing atoms for adsorption, and $f_{\text {prist }}^{0}$ is the resonance frequency when not dosing atoms and keeping the nanotube pristine. The constant $A$ is introduced to account for variations in the spring constant between the measurement of $f_{\text {ads }}^{0}(T)$ and that of $f_{\text {prist }}^{0}(T)$; indeed, the spring constant can be different, if for instance the gate voltage applied in the measurement of $f_{\text {ads }}^{0}(T)$ differs from that of $f_{\text {prist }}^{0}(T)$ (Supplemental Material, Sec. IV [16]). The constant $A$ is fixed so that $\varphi=0$ at high $T$. In Eq. (1), we assume that the spring tension is insensitive to the tension induced by the interaction between noble gas atoms, which is 2 orders of magnitude weaker than that of covalent C-C bonds [7].

Figure 2(a) shows the temperature dependence of the coverage while dosing $\mathrm{Kr}$ atoms. Above a characteristic 
(a)

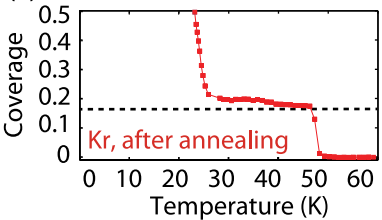

(b)

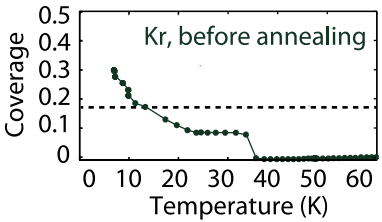

FIG. 2 (color online). (a) Coverage upon dosing $\mathrm{Kr}$ atoms while lowering the temperature $T$ with a ramping rate $0.016 \mathrm{~K} / \mathrm{s}$. (b) Same measurement recorded before having current annealed the nanotube with a $T$ ramping rate $0.033 \mathrm{~K} / \mathrm{s}$. The pressure is $3 \times 10^{-7}$ mbar for both measurements.

temperature $T_{c} \simeq 48 \mathrm{~K}$, the coverage remains at zero. On lowering temperature, the coverage jumps to $\varphi \simeq 1 / 6$ and remains close to this value until $T \simeq 26 \mathrm{~K}$. This behavior can be accounted for by the balance of atoms impinging on and departing from the nanotube. For $T>T_{c}$, an impinging atom departs very rapidly from the nanotube, so that the number of adsorbed atoms remains close to zero [Fig. 1(e)]. For $T<T_{c}$, it is energetically favorable for the atoms to stay on the nanotube [Fig. 1(f)] - the atoms forming a layer with $\varphi \simeq 1 / 6$. This layer is likely a monolayer, because the coverage $\varphi \simeq 1 / 6$ of $\mathrm{Kr}$ on graphite corresponds to a monolayer (Ref. [13], Chap. 6). Upon further lowering temperature so that $T \ll T_{c}$, the coverage gets larger than $\varphi=1 / 6$, indicating that $\mathrm{Kr}$ atoms start to form the second layer. The coverage grows in a monotonic way without any additional plateaus even when the coverage gets larger than one (Supplemental Material, Sec. VII [16]). The absence of additional plateaus above $\varphi=1 / 6$ further supports the interpretation of the coverage $\varphi \simeq 1 / 6$ as being related to the monolayer.

Key to this work is annealing the nanotube by passing a large current through it. After the measurements shown in Fig. 2(a), we exposed the nanotube to ambient air. We then baked the cryostat and the nanotube at $110^{\circ} \mathrm{C}$ under vacuum for two days to reach a base pressure of $\sim 3 \times 10^{-11}$ mbar. We again measured the coverage upon lowering $T$ while dosing $\mathrm{Kr}$ atoms. Figure 2(b) shows that $T_{c}$ is much lower than before, and the coverage at $T \lesssim T_{c}$ is significantly lower than $1 / 6$. We had to anneal the nanotube with a current of $\sim 10 \mu \mathrm{A}$ in order to recover the same measurement as in Fig. 2(a). These measurements suggest that the growth of monolayers is extremely sensitive to contamination, since a simple exposure to air prevents the formation of homogeneous monolayers. Another advantage of current annealing is that it brings the nanotube back to its pristine state after the adsorption of noble gas atoms on its surface.

We grew different monolayers on the nanotube by dosing $\mathrm{Xe}, \mathrm{Kr}, \mathrm{Ar}$, and $\mathrm{Ne}$ (Fig. 3). The nanotube surface was cleaned by current annealing before each growth. Upon decreasing temperature, the coverage increases rapidly from zero to a plateau with $\varphi \simeq 1 / 6$, indicating the growth of the monolayer. The characteristic temperature of the monolayer growth depends on the atomic species; $T_{c}$ is higher when the atomic mass is larger (Fig. 3). We attribute the origin of the variation of $T_{c}$ to the polarizability of the atomic species and the van der Waals interaction between the atom and the nanotube, the polarizability and the interaction both increasing with the atomic radius. We also carried out experiments where we evaporated the monolayers from the nanotube by continuously increasing the temperature of the cryostat from 4 to $\sim 100 \mathrm{~K}$. The coverage jumped from $\simeq 1 / 6$ to 0 at a temperature that is up to $\sim 10 \mathrm{~K}$ higher than $T_{c}$ (Supplemental Material, Sec. V [16]).

We measured the time of the growth of monolayers from $\varphi=0$ to $\varphi=1 / 6$ while keeping the temperature constant. This time gets longer for lower pressure (Supplemental Material, Sec. VI [16]).

Xenon monolayers are particularly robust against temperature changes. Figure 4(a) shows coverage-temperature measurements recorded at different pressures and different temperature ramping rates. $T_{c}$ varies from one

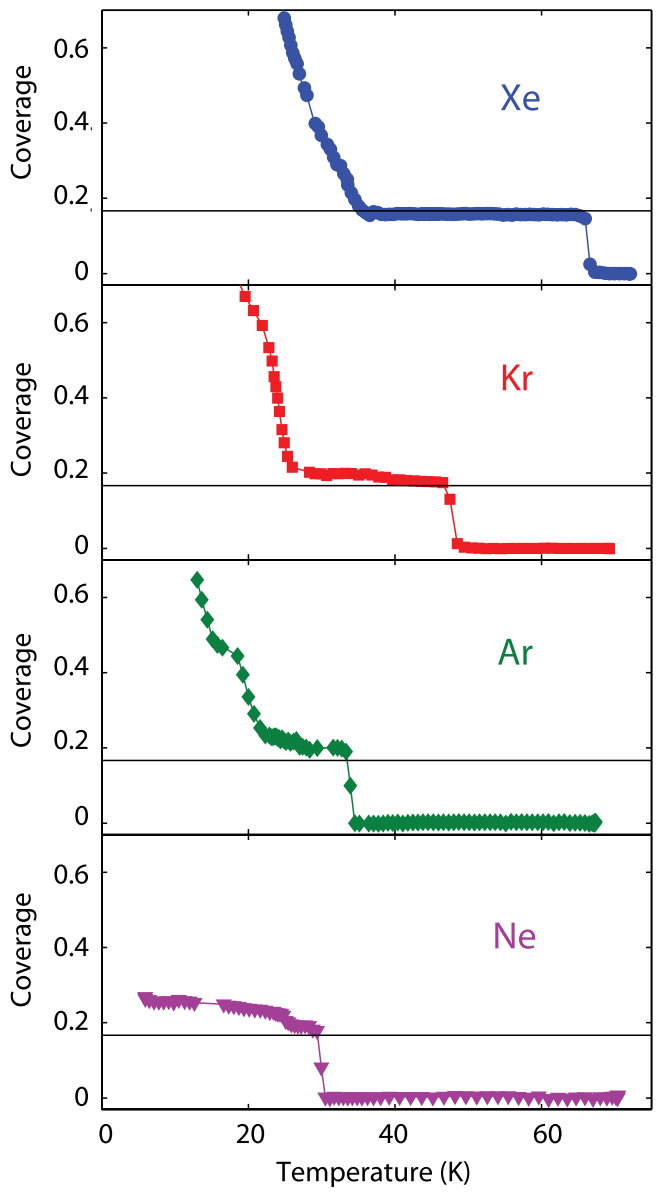

FIG. 3 (color online). Coverage upon lowering temperature while dosing $\mathrm{Xe}, \mathrm{Kr}, \mathrm{Ar}$, and $\mathrm{Ne}$ atoms. The pressure is $3 \times$ $10^{-7} \mathrm{mbar}$ for all measurements. The $T$ ramping rate is $0.008 \mathrm{~K} / \mathrm{s}$ for the Xe measurement and $0.016 \mathrm{~K} / \mathrm{s}$ for the other measurements. The black line corresponds to $\varphi=1 / 6$. 
measurement to the next. However, the plateau in coverage at $1 / 6$ is clearly reproducible. This shows that Xe monolayers are energetically stable with the number of atoms being insensitive to temperature over a large parameter space. In contrast, measurements with $\mathrm{Ne}$ feature a plateau whose coverage depends significantly on $T$ [Fig. 4(b)].

We now discuss the nature of the monolayers of $\mathrm{Xe}, \mathrm{Kr}$, Ar, and Ne. For this, we carried out theoretical calculations to predict whether the solid phases are commensurate or incommensurate in the limit of zero temperature. In addition, we estimated the melting temperature of the different solid phases. To this end, we performed a series of Monte Carlo simulations relying on standard interatomic potentials between noble gas atoms and the carbon atoms of the nanotube (Supplemental Material, Sec. IX [16]). This microscopic study was carried out for nanotubes with diameters in the range of 21-38 A, which covers the typical diameters obtained with our chemical vapor deposition recipe.

Our experimental findings indicate that Xe monolayers are commensurate solids. First, the coverage of the monolayer is $1 / 6$. Second, the coverage remains at this value over a large temperature range. This robustness suggests that Xe atoms are strongly bound to the underlying carbon surface, as is the case for commensurate solids. Our experimental results are accounted for by our theoretical calculations, which predict that the solid is a registered $\sqrt{3} \times \sqrt{3}$ crystal at zero temperature. Moreover, this solid phase is calculated to become unstable at $\sim 80 \mathrm{~K}$, which is consistent with the melting temperature measured in Fig. S4 of the Supplemental Material [16].

Monolayers of $\mathrm{Ar}$ and $\mathrm{Ne}$ are less stable, since the measured coverage depends significantly on temperature for $T \lesssim T_{c}$. Our calculations reveal that, in the limit of zero temperature, $\mathrm{Ar}$ and $\mathrm{Ne}$ monolayers are incommensurate solids with coverages 0.265 and 0.403 , respectively. The measured coverages at $T \lesssim T_{c}$ are much lower than these predicted values, suggesting that the monolayers observed experimentally are not in the solid phase. Moreover, our calculations show that incommensurate
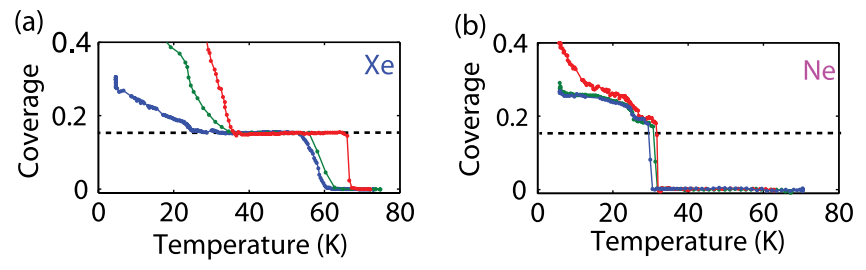

FIG. 4 (color online). (a) Coverage upon lowering temperature while dosing $\mathrm{Xe}$ atoms. The pressure is $7 \times 10^{-8}, 3 \times 10^{-7}$, and $3 \times 10^{-7} \mathrm{mbar}$, and the $T$ ramping rate is $0.016,0.033$, and $0.008 \mathrm{~K} / \mathrm{s}$ for the blue, green, and red lines, respectively. (b) Coverage upon lowering temperature while dosing Ne atoms. The pressure is $3 \times 10^{-7} \mathrm{mbar}$ for all three measurements, and the $T$ ramping rate is $0.016 \mathrm{~K} / \mathrm{s}$ for the blue and the green lines and $0.008 \mathrm{~K} / \mathrm{s}$ for the red line. solids melt at temperatures as low as $5 \mathrm{~K}$ when the coverage is set to the values we typically measure at $T \lesssim T_{c}$. This further indicates that the monolayers of $\mathrm{Ar}$ and $\mathrm{Ne}$, observed experimentally at $25-35 \mathrm{~K}$, are in the liquid phase.

As for $\mathrm{Kr}$, the measured temperature dependence of the coverage is similar to that of Xe, supporting the scenario that $\mathrm{Kr}$ monolayers are commensurate solid phases. This result would be in agreement with experimental signatures of the stability of a commensurate $\mathrm{Kr}$ layer on graphite up to quite high temperatures, $T \sim 130 \mathrm{~K}$ [25]. However, the coverage of $\mathrm{Kr}$ slightly depends on temperature in the plateau region (Fig. 3), showing that $\mathrm{Kr}$ monolayers are less pinned to the carbon surface than Xe monolayers. Previous theoretical calculations of the $\mathrm{Kr}$ monolayer on graphite show that corrugation effects are extremely important to get the $\sqrt{3} \times \sqrt{3}$ crystal stable [26,27]. Only by increasing in an empirical way the anisotropic part of the pair interaction, the commensurate phase becomes stable. Our present simulations on $\mathrm{Kr}$ adsorbed on nanotubes show the same trend. Therefore, more work is needed to establish the phase of $\mathrm{Kr}$ monolayers on nanotubes.

To conclude, we studied the formation of noble gas atom monolayers on individual nanotubes. We found that Xe atoms form robust commensurate solids, whereas Ar and $\mathrm{Ne}$ atoms organize themselves in fluids. These monolayers consist of $\sim 10^{5}$ atoms, which is a tiny amount of material difficult to detect with most experimental techniques used in surface science. The study of these monolayers was possible here because nanotube mechanical resonators are extremely sensitive probes. The second important aspect of our experiments is that the nanotube surface was ultraclean; this was achieved by thoroughly current annealing the nanotube in ultrahigh vacuum. These resonators made from ultraclean nanotubes are promising for various future adsorption experiments, such as the measurement of new phase transitions emerging in the one-dimensional limit with narrower nanotubes, the investigation of quantum effects of $\mathrm{He}$ monolayers adsorbed on nanotubes [28], the study of the diffusion of adsorbed atoms over the resonator surface which is a topic of increasing interest [29], and the interplay between the strong mechanical nonlinearities of nanotubes [14,30-33] and the diffusion of atoms $[34,35]$.

We thank A. Isacsson and J. Moser for discussions. We acknowledge support from the European Union through the Graphene Flagship (Grant No. 604391), the ERCcarbonNEMS project, and a Marie Curie Grant (No. 271938), the Spanish state (Grant No. MAT201231338), and the Catalan government (AGAUR, SGR). C. G. and J. B. acknowledge partial financial support from the Junta de de Andalucía Group PAI-205, Grant No. FQM5987, MICINN (Spain) Grants No. FIS2010-18356 and No. FIS2011-25275, and Generalitat de Catalunya Grant No. 2009SGR-1003. 
*Present address: CNRS, Laboratoire de Photonique et de Nanostructures, UPR20, route de Nozay, 91460 Marcoussis, France

†Present address: Department of Physics, ETH Zurich, Schafmattstrasse 16, 8093 Zurich, Switzerland.

[1] A. Dillon, K. Jones, T. Bekkedahl, C. Kiang, D. Bethune, and M. Heben, Nature (London) 386, 377 (1997).

[2] W. Teizer, R. B. Hallock, E. Dujardin, and T. W. Ebbesen, Phys. Rev. Lett. 82, 5305 (1999).

[3] H. Ulbricht, J. Kriebel, G. Moos, and T. Hertel, Chem. Phys. Lett. 363, 252 (2002).

[4] W. Shi and J. K. Johnson, Phys. Rev. Lett. 91, 015504 (2003).

[5] J.-C. Lasjaunias, K. Biljaković, J.-L. Sauvajol, and P. Monceau, Phys. Rev. Lett. 91, 025901 (2003).

[6] H. Ulbricht, R. Zacharia, N. Cindir, and T. Hertel, Carbon 44, 2931 (2006).

[7] Z. Wang, J. Wei, P. Morse, J. G. Dash, O. E. Vilches, and D. H. Cobden, Science 327, 552 (2010).

[8] H.-C. Lee, O. E. Vilches, Z. Wang, E. Fredrickson, P. Morse, R. Roy, B. Dzyubenko, and D. H. Cobden, J. Low Temp. Phys. 169, 338 (2012).

[9] J. Chaste, A. Eichler, J. Moser, G. Ceballos, R. Rurali, and A. Bachtold, Nat. Nanotechnol. 7, 301 (2012).

[10] H.-Y. Chiu, P. Hung, H. W. C. Postma, and M. Bockrath, Nano Lett. 8, 4342 (2008).

[11] B. Lassagne, D. Garcia-Sanchez, A. Aguasca, and A. Bachtold, Nano Lett. 8, 3735 (2008).

[12] K. Jensen, K. Kim, and A. Zettl, Nat. Nanotechnol. 3, 533 (2008).

[13] L. W. Bruch, M. W. Cole, and E. Zaremba, Physical Adsorption: Forces and Phenomena (Clarendon Press, Oxford, 1997).

[14] A. Eichler, J. Moser, J. Chaste, M. Zdrojek, I. Wilson-Rae, and A. Bachtold, Nat. Nanotechnol. 6, 339 (2011).

[15] A. K. Huttel, G. A. Steele, B. Witkamp, M. Poot, L. P. Kouwenhoven, and H. S. van der Zant, Nano Lett. 9, 2547 (2009).

[16] See Supplemental Material, which includes Refs. [17-22], at http://link.aps.org/supplemental/10.1103/PhysRevLett
.112 .196103 for additional information on the sample fabrication and the experimental setup, further measurements, and the theoretical results of the microscopic simulation of the adsorption of noble gas atoms on nanotubes.

[17] C. Zhou, J. Kong, and H. Dai, Phys. Rev. Lett. 84, 5604 (2000).

[18] L. Bruch and J. Venables, Surf. Sci. 148, 167 (1984).

[19] A. Cheng and M. L. Klein, Langmuir 8, 2798 (1992).

[20] W. A. Steele, J. Phys. Chem. 82, 817 (1978).

[21] M. L. Klein, S. O'Shea, and Y. Ozaki, J. Phys. Chem. 88, 1420 (1984).

[22] H.-Y. Kim, M. W. Cole, M. Mbaye, and S. M. Gatica, J. Phys. Chem. A 115, 7249 (2011).

[23] V. Gouttenoire, T. Barois, S. Perisanu, J.-L. Leclercq, S. T. Purcell, P. Vincent, and A. Ayari, Small 6, 1060 (2010).

[24] J. Chaste, M. Sledzinska, M. Zdrojek, J. Moser, and A. Bachtold, Appl. Phys. Lett. 99, 213502 (2011).

[25] E. D. Specht, A. Mak, C. Peters, M. Sutton, R. J. Birgeneau, D. L. D'Amico, D. E. Moncton, S. E. Nagler, and P. M. Horn, Z. Phys. B 69, 347 (1987).

[26] G. Vidali and M. W. Cole, Phys. Rev. B 29, 6736 (1984).

[27] N. D. Shrimpton and W. A. W. A. Steele, Phys. Rev. B 44, 3297 (1991).

[28] M. C. Gordillo and J. Boronat, Phys. Rev. B 86, 165409 (2012).

[29] Y. Yang, C. Callegari, X. Feng, and M. Roukes, Nano Lett. 11, 1753 (2011).

[30] A. Eichler, M. del Álamo Ruiz, J. A. Plaza, and A. Bachtold, Phys. Rev. Lett. 109, 025503 (2012).

[31] A. Eichler, J. Moser, M. Dykman, and A. Bachtold, Nat. Commun. 4, 2843 (2013).

[32] H. B. Meerwaldt, G. Labadze, B. H. Schneider, A. Taspinar, Y. M. Blanter, H. S. J. van der Zant, and G. A. Steele, Phys. Rev. B 86, 115454 (2012).

[33] A. W. Barnard, V. Sazonova, A. M. van der Zande, and P. L. McEuen, Proc. Natl. Acad. Sci. U.S.A. 109, 19093 (2012).

[34] J. Atalaya, A. Isacsson, and M. I. Dykman, Phys. Rev. Lett. 106, 227202 (2011).

[35] J. Atalaya, A. Isacsson, and M. I. Dykman, Phys. Rev. B 83, 045419 (2011). 